\title{
Effect of heat-flux boundary conditions on the Rayleigh-Bénard instability in a rarefied gas
}

\author{
Y. Ben-Ami and A. Manela \\ Faculty of Aerospace Engineering, Technion - Israel Institute of Technology, Haifa 32000, Israel
}

(Received 25 October 2018; published 14 March 2019)

\begin{abstract}
We consider the effect of heat-flux boundary conditions, replacing the previously studied isothermal wall conditions, on the Rayleigh-Bénard instability in a rarefied gas. The problem is investigated in the limit of small Knudsen numbers, by means of a linear stability analysis of a slip flow model, and the direct simulation Monte Carlo method. In the latter, a noniterative algorithm is applied to implement the heat-flux conditions. The results delineate the instability domain in the parameters plane of the Knudsen number $(\mathrm{Kn})$, Froude number $(\mathrm{Fr})$, and walls reference temperature ratio. The heat-flux conditions result in a significant destabilizing effect, extending instability to larger Knudsen numbers. At large Fr, the Boussinesq limit is recovered, and transition to instability is governed by a critical value of the Rayleigh number. With decreasing Fr, gas compressibility becomes dominant, confining the convection layer to the vicinity of the upper cold wall. Asymptotic analysis of the low-Fr limit is carried out, to highlight the impact of difference in thermal conditions. The Monte Carlo scheme is applied to investigate system instability at supercritical states, where walls heat-flux conditions lead to elevated shear stresses. Nonmonotonic variations in the walls shear stress with $\mathrm{Kn}$ are observed and discussed.
\end{abstract}

DOI: 10.1103/PhysRevFluids.4.033402

\section{INTRODUCTION}

The Rayleigh-Bénard (RB) problem, investigating the thermally driven convection formed in a fluid confined between parallel horizontal plates and heated from below, is a classical problem in hydrodynamic stability [1,2]. Relevant in the analysis of various geophysical, atmospheric, and astrophysical phenomena, the problem has been studied extensively over the years, combining theoretical, numerical, and experimental investigations. Existing works have spanned a variety of topics in the RB problem, covering the linear and nonlinear regimes with different geometrical setups and fluid characteristics (see, e.g., the review in Ref. [3], and references cited therein). Nonetheless, most of these works have been carried out in the framework of the Boussinesq approximation, where fluid density variations are allowed only due to thermal expansion, and the impact of gas compressibility is neglected. Under this assumption, the only governing parameter for the onset of instability is the Rayleigh number, representing a balance between fluid buoyancy, heat conductivity, and viscosity effects [1].

While the Boussinesq approximation holds in a large number of cases of practical importance, it becomes invalid in states where flow compressibility is nonnegligible. Such scenarios evolve in the study of RB convection in rarefied gases, where the effect of gas potential energy becomes comparable to its internal energy. The problem has been studied during the past few decades, combining numerical and analytical approaches to characterize the system response at subcritical, critical, and supercritical conditions [4-11]. The viscous-compressible problem is governed, apart from the cold to hot walls temperature ratio, by the Knudsen number, marking the proportion between the molecular mean free path and the boundaries separation distance, and the Froude number, representing the ratio between fluid inertia and buoyancy. As established by Stefanov et al. 
[6], rarefied gas RB instability is confined to the continuum limit of small Knudsen numbers even when large walls temperature differences are imposed. Later studies (e.g., Ref. [8]) have shown that the dampening effect of gas rarefaction results from the combined impacts of gas viscosity (dominant at large Froude numbers) and compressibility (at $O(1)$ Froude numbers), analyzing the specific characteristics of transition to instability at non-Boussinesq conditions.

All of the above RB studies in rarefied gases have focused on a setup where the walls temperatures are fixed. It is nevertheless of fundamental and practical importance to examine the impact of different forms of the thermal boundary conditions on the system stability. Within the Boussinesq approximation, this problem has been considered by replacing the isothermal with heat-flux wall conditions, and a significant destabilizing effect of the latter has been observed [12-15]. Specifically, Sparrow et al. [12] showed that the critical Rayleigh number for instability is reduced when heat-flux boundary conditions are imposed. Jakeman [13] has then demonstrated that in this case larger convection cells are formed, pointing to smaller critical perturbation wave numbers. Indeed, transition to instability in the walls fixed heat-flux setup is characterized by infinitely long waves, in marked difference from the finite-wavelength instability occurring in the counterpart Boussinesq isothermal walls problem.

In light of the contradicting stabilizing and destabilizing effects of gas rarefaction and heat-flux boundary conditions on the RB instability, it appears of interest to study the impact of heat-flux conditions on the RB instability in a rarefied gas. Focusing on the near-continuum limit, the present work analyzes the problem both numerically, using the direct simulation Monte Carlo (DSMC) method, and theoretically, based on linear stability analysis of the viscous-compressible NavierStokes-Fourier equations. The results obtained are discussed in view of existing studies on the walls temperature-fixed problem, to asses the specific impact of the difference in boundary conditions. In Sec. II, the problem is stated and analyzed. The threshold of instability is discussed in Sec. III, and a specific investigation of the limit of dominant compressibility is made in Sec. IV. The nonlinear problem at supercritical conditions is considered in Sec. V, followed by conclusions in Sec. VI.

\section{PROBLEM STATEMENT AND ANALYSIS}

Consider a layer of a perfect monatomic gas of average density $\rho_{0}^{*}$ confined between two horizontal fully diffuse walls at $x_{2}^{*}=\mp D^{*} / 2$. The gas is subject to a gravity force of magnitude $g^{*}$ per unit mass in the negative vertical direction, $\mathbf{g}^{*}=-g^{*} \hat{\mathbf{x}}_{\mathbf{2}}$, and is heated with a normal heat-flux,

$$
\mathbf{q}_{\mathbf{w}}^{*}\left(\mp D^{*} / 2\right)=q_{w}^{*} \hat{\mathbf{x}}_{\mathbf{2}} \text {. }
$$

By inserting and removing the same amount of heat at the walls, the total thermal energy of the system,

$$
E_{\mathrm{th}}^{*}=\rho_{0}^{*} c_{v}^{*} T_{0}^{*},
$$

is conserved. In Eq. (2), $c_{v}^{*}$ denotes the gas specific heat capacity at constant volume, and $T_{0}^{*}$ is the uniform gas temperature obtained at $q_{w}^{*}=0$.

To render the formulation dimensionless, the position vector is normalized by the layer width $D^{*}$, the gas density by its mean value $\rho_{0}^{*}$, and the temperature by $T_{h}^{*}$, the reference temperature of the lower hot wall. While the hot $\left(T_{h}^{*}\right)$ and cold $\left(T_{c}^{*}\right)$ walls temperatures are not imposed in the present setup, the scaling by $T_{h}^{*}$ is introduced to facilitate comparison between the heat-flux and the temperature-prescribed problems. The equivalence between the formulations in terms of the reference "pure conduction" state is considered in Sec. II A, where the correlation between the $\left(q_{w}^{*}, T_{0}^{*}\right)$ and $\left(T_{h}^{*}, T_{c}^{*}\right)$ prescriptions is specified. Making use of the $T_{h}^{*}$ scale, the gas velocity is nondimensionalized using the mean thermal speed $U_{\mathrm{th}}^{*}=\sqrt{2 \mathcal{R}^{*} T_{h}^{*}}$, where $\mathcal{R}^{*}$ denotes the specific gas constant. The time is then normalized by $D^{*} / U_{\mathrm{th}}^{*}$, the pressure by $\rho_{0}^{*} U_{\mathrm{th}}^{* 2}$, and the heat-flux by $\rho_{0}^{*} U_{\mathrm{th}}^{* 3}$. The transport coefficients of shear viscosity and heat conductivity are normalized by their respective values $\mu_{h}^{*}=\rho_{0}^{*} U_{\mathrm{th}} D^{*}$ and $\kappa_{h}^{*}=c_{v}^{*} \rho_{0}^{*} U_{\mathrm{th}} D^{*}$ at $T_{h}^{*}$, respectively. 
Focusing on the continuum limit of small Knudsen numbers, the gas state is governed by a "slip flow" model consisting of the Navier-Stokes-Fourier equations of continuity, momentum and energy, together with the equation of state for a perfect gas. These are supplemented by the impermeability, velocity slip, and heat-flux boundary conditions, together with normalization conditions fixing the total mass and thermal energy of the system. Applying the above scaling, the normalized equations for the gas density $\rho$, velocity vector $\mathbf{u}=\left(u_{1}, u_{2}, u_{3}\right)$, pressure $p$, and temperature $T$ are

$$
\begin{aligned}
& \frac{\partial \rho}{\partial t}+\nabla \cdot(\rho \mathbf{u})=0, \\
& \left.\rho \frac{D \mathbf{u}}{D t}=-\nabla p+\mathrm{Kn} \nabla \cdot\left[2 \mu\left(\mathrm{S}-\frac{1}{3} \nabla \cdot \mathbf{u}\right) \mid\right)\right]-\frac{\rho}{\mathrm{Fr}} \hat{\mathbf{x}}_{\mathbf{2}}, \\
& \rho \frac{D T}{D t}=\mathrm{Kn} \nabla \cdot(\kappa \nabla T)-(\gamma-1) p \nabla \cdot \mathbf{u}+2(\gamma-1) \operatorname{Kn} \Phi,
\end{aligned}
$$

and

$$
p=\frac{1}{2} \rho T
$$

In the above, $D / D t$ denotes the material derivative, I marks the identity tensor, $\mathrm{S}=$ $\left((\nabla \mathbf{u})+(\nabla \mathbf{u})^{\mathrm{T}}\right) / 2$ is the rate of strain tensor, and $\Phi=2 \mu\left(\mathrm{S}: \mathrm{S}-(\boldsymbol{\nabla} \cdot \mathbf{u})^{2} / 3\right)$ is the rate of viscous dissipation. We consider a monoatomic hard-sphere gas, for which the Chapman-Enskog scheme yields

$$
\mu(T)=\frac{5 \sqrt{\pi}}{16} T^{1 / 2} \quad \text { and } \quad \kappa(T)=\frac{5 \sqrt{\pi}}{16} \frac{\gamma}{\operatorname{Pr}} T^{1 / 2}
$$

for the nondimensional transport coefficients of shear viscosity $\mu$ and heat conductivity $\kappa$, respectively [16]. The dimensionless parameters appearing in Eqs. (4), (5), and (7) are the Knudsen number, $\mathrm{Kn}=l^{*} / D^{*}$, where $l^{*}$ denotes the mean-free path of a gas molecule; the Froude number, $\mathrm{Fr}=U_{\mathrm{th}}^{* 2} / g^{*} D^{*}$; the ratio of specific heats, $\gamma=c_{p}^{*} / c_{v}^{*}$, where $c_{p}^{*}$ marks the gas specific heat at constant pressure; and the Prandtl number, $\operatorname{Pr}=\mu_{h}^{*} c_{p}^{*} / \kappa_{h}^{*}$. For a monatomic hard-sphere gas model considered hereafter, $\gamma=5 / 3$ and $\operatorname{Pr}=2 / 3$ [16].

The above equations are supplemented by the normalization conditions

$$
\int_{V} \rho d V=1 \text { and } \int_{V} \rho T d V=T_{0},
$$

where $T_{0}=T_{0}^{*} / T_{h}^{*}$, fixing the system total mass and energy at the gas volume $V$, respectively, and the boundary conditions

$$
u_{1}= \pm \zeta \frac{\partial u_{1}}{\partial x_{2}}+\sigma \frac{\partial T}{\partial x_{1}}, \quad u_{2}=0 \quad \text { and } \quad-\frac{3 \mathrm{Kn}}{4} \kappa(T) \frac{\partial T}{\partial x_{2}}=q_{w} \quad \text { at } \quad x_{2}=\mp \frac{1}{2},
$$

imposing gas impermeability and specifying the magnitudes of velocity-slip and heat-flux at the walls. Here, $\zeta=1.254 \sqrt{\pi} \mathrm{Kn} / 2$ and $\sigma=0.646 \sqrt{\pi} \mathrm{Kn} / 2$ are the first-order coefficients of velocity slip and thermal creep for a hard-sphere gas [16], respectively. Strictly, the slip condition in Eq. (9) was derived for a diffuse reflecting boundary with fixed temperature, and its compatibility with the present heat-flux condition requires rigorous examination. As such a study was not carried out previously, we assign the present condition for convenience and rely on the agreement between the DSMC and continuum-limit predictions (see Sec. III) to justify its application. Additionally, it should be noted that the slip condition in Eq. (9) was derived for a "slightly compressible" gas, and not for the full compressible Navier Stokes equations studied hereafter. Investigation of the latter was carried out in a recent contribution by Aoki et al. [17], where the slip and jump conditions were obtained for a temperature-fixed surface, taking into account the effect of density variations near the surface on the local mean free path. As these conditions, as in Eq. (9), do not apply for a fixed 
heat-flux boundary, and their application do not yield visible differences in the results in the current small-Kn setup, we retain Eq. (9) for simplicity.

\section{A. The reference state}

The reference "pure conduction" state is obtained by setting $\mathbf{u}=\mathbf{0}$ in Eqs. (3)-(5) and integrating over the $x_{2}$-momentum and energy balances. Making use of the heat-flux condition in Eq. (9), this yields

$$
T^{(0)}=\left(-A x_{2}+B\right)^{2 / 3} \quad \text { and } \quad \rho^{(0)}=\frac{C}{T^{(0)}} \exp \left(\frac{6}{A \mathrm{Fr}} \sqrt{T^{(0)}}\right),
$$

where the superscript " $(0)$ " denotes a reference state, $A=64 q_{w} /(25 \sqrt{\pi} \mathrm{Kn})$, and the constants $B$ and $C$ are fixed via Eq. (8). To facilitate comparison between the present and the walls fixed-temperature problem, it is convenient to relate the above calculation of coefficients to the expressions obtained by application of the temperature-jump boundary conditions,

$$
T=1+\tau \frac{\partial T}{\partial x_{2}} \quad \text { at } \quad y=-\frac{1}{2} \quad \text { and } \quad T=R_{T}-\tau \frac{\partial T}{\partial x_{2}} \quad \text { at } \quad y=\frac{1}{2} .
$$

Here, $R_{T}=T_{c}^{*} / T_{h}^{*}$ is the walls cold to hot reference temperature ratio, and $\tau=2.4 \sqrt{\pi} \mathrm{Kn} / 2$ is the first-order temperature jump coefficient for a hard-sphere gas [16]. Substitution of $T^{(0)}$ in Eq. (10) into Eq. (11) yields a pair of coupled algebraic equations for the constants $A$ and $B$, which are solved numerically. Having determined $A$ and $B$, the normalization constants $C$ and $T_{0}$ are obtained using Eq. (8), to yield

$$
\begin{aligned}
C= & \frac{2}{\operatorname{Fr}\left\{\exp \left[\frac{6}{A F r}\left(\frac{1}{2} A+B\right)^{1 / 3}\right]-\exp \left[\frac{6}{A F r}\left(-\frac{1}{2} A+B\right)^{1 / 3}\right]\right\}} \text { and } \\
T_{0}= & \frac{C \operatorname{Fr}}{36}\left\{\left[(A F r)^{2}-6(A F r)\left(\frac{1}{2} A+B\right)^{1 / 3}+18\left(\frac{1}{2} A+B\right)^{2 / 3}\right] \exp \left[\frac{6}{A F r}\left(\frac{1}{2} A+B\right)^{1 / 3}\right]\right. \\
& \left.-\left[(A F r)^{2}-6(A F r)\left(-\frac{1}{2} A+B\right)^{1 / 3}+18\left(-\frac{1}{2} A+B\right)^{2 / 3}\right] \exp \left[\frac{6}{A F r}\left(-\frac{1}{2} A+B\right)^{1 / 3}\right]\right\} .
\end{aligned}
$$

This elucidates the equivalence, in terms of the reference-state problem formulations, between the impositions of walls heat-flux (where $q_{w}$ and $T_{0}$ are fixed) and walls temperatures (where the walls temperatures $T_{h}=1$ and $T_{c}=R_{T}$ are prescribed). This equivalence facilitates the comparison between the stability properties of the walls-prescribed heat-flux and temperature systems.

\section{B. Linear stability analysis}

The stability of the reference state is studied by means of a linear temporal stability analysis. Toward this end, each of the hydrodynamic fields is represented by the sum of its reference value and a small spatially harmonic perturbation,

$$
F \approx F^{(0)}\left(x_{2}\right)+\varepsilon \phi^{(1)}\left(x_{2}\right) \exp [\text { ik } \cdot \mathbf{r}+\omega t]
$$

where $\varepsilon \ll 1$. Also appearing in Eq. (13) are $\mathbf{k}$ and $\mathbf{r}$, marking the wave number and position vectors in the $\left(x_{1}, x_{3}\right)$ plane parallel to the walls, respectively, and the complex number $\omega=\omega_{r}+i \omega_{i}$ denoting the perturbation growth rate $\omega_{r}$ and frequency $\omega_{i}$. The problem is transversely symmetric about the $x_{2}$ axis, and we may therefore, with no loss of generality, confine our analysis to planes normal to the $\mathbf{k} \times \hat{\mathbf{x}}_{\mathbf{2}}$ direction. Redefining our system of coordinates so that its $x$ axis is parallel to $\mathbf{k}$ and $u$ and $v$ are the flow components in the $k$ and normal $y$ (formerly $x_{2}$ ) directions, respectively, the notation is further simplified by denoting $f=i k u$. Substituting Eq. (13) into Eqs. (3)-(5), neglecting 
nonlinear terms, and eliminating the pressure $p$ by use of the equation of state Eq. (6), we obtain the linearized perturbation problem consisting of

$$
\begin{aligned}
\omega \rho^{(1)}= & -\rho^{(0)}\left(f^{(1)}+\frac{d v^{(1)}}{d y}\right)-v^{(1)} \frac{d \rho^{(0)}}{d y}, \\
\omega \rho^{(0)} f^{(1)}= & \frac{1}{2} k^{2}\left(\rho^{(0)} T^{(1)}+T^{(0)} \rho^{(1)}\right)+\operatorname{Kn}\left\{\mu^{(0)}\left[\frac{d^{2} f^{(1)}}{d y^{2}}-\frac{k^{2}}{3}\left(\frac{d v^{(1)}}{d y}+4 f^{(1)}\right)\right]\right. \\
& \left.+\frac{d \mu^{(0)}}{d y}\left[\frac{d f^{(1)}}{d y}-k^{2} v^{(1)}\right]\right\}, \\
\omega \rho^{(0)} v^{(1)}= & -\frac{1}{2}\left[\rho^{(0)} \frac{d T^{(1)}}{d y}+T^{(1)} \frac{d \rho^{(0)}}{d y}+T^{(0)} \frac{d \rho^{(1)}}{d y}+\rho^{(1)}\left(\frac{d T^{(0)}}{d y}+\frac{2}{\mathrm{Fr}}\right)\right] \\
& +\operatorname{Kn}\left\{\mu^{(0)}\left[\frac{4}{3} \frac{d^{2} v^{(1)}}{d y^{2}}+\frac{1}{3} \frac{d f^{(1)}}{d y}-k^{2} v^{(1)}\right]+\frac{2}{3} \frac{d \mu^{(0)}}{d y}\left[2 \frac{d v^{(1)}}{d y}-f^{(1)}\right]\right\},
\end{aligned}
$$

and

$$
\begin{aligned}
\omega \rho^{(0)} T^{(1)}= & -\rho^{(0)} v^{(1)} \frac{d T^{(0)}}{d y}-\frac{2}{3} \rho^{(0)} T^{(0)}\left(f^{(1)}+\frac{d v^{(1)}}{d y}\right)+\operatorname{Kn}\left\{\left[\frac{d^{2} \kappa^{(0)}}{d y^{2}}-k^{2} \kappa^{(0)}\right] T^{(1)}\right. \\
& \left.+2 \frac{d \kappa^{(0)}}{d y} \frac{d T^{(1)}}{d y}+\kappa^{(0)} \frac{d^{2} T^{(1)}}{d y^{2}}\right\}
\end{aligned}
$$

where $\mu^{(0)}=(5 \sqrt{\pi} / 16) \sqrt{T^{(0)}}$ and $\kappa^{(0)}=(25 \sqrt{\pi} / 32) \sqrt{T^{(0)}}$, in accordance with Eq. (7). The problem is supplemented by the velocity-slip boundary conditions,

$$
f^{(1)}= \pm \zeta \frac{d f^{(1)}}{d y}-\sigma k^{2} T^{(1)} \quad \text { at } \quad y=\mp \frac{1}{2}
$$

as well as adiabatic boundary conditions for the walls heat-flux perturbation,

$$
2 T^{(0)} \frac{d T^{(1)}}{d y}+\frac{d T^{(0)}}{d y} T^{(1)}=0 \quad \text { at } \quad y=\mp \frac{1}{2} .
$$

The harmonic form in Eq. (13) for the density and temperature perturbations identically satisfies the homogeneous counterparts of the mass and energy normalization conditions in Eq. (8).

Comparing between the present and walls fixed-temperature formulations, it is observed that, while the reference-state description is unaffected by the difference in boundary conditions, the linear stability problems differ in the forms of perturbations wall conditions. In contrast with the isothermal-walls setup, the adiabatic conditions result in variations in the walls temperatures, subsequently not retaining their reference $R_{T}$ ratio. As shown below, this modification has a significant impact on the system stability characteristics.

To solve the linear stability problem, the dispersion relation for the system eigenvalue $\omega$ is calculated by means of the Chebyshev collocation method [18]. Application of this method yields an algebraic eigenvalue problem consisting of $4 \mathrm{~N}$ coupled linear equations for the perturbations $\rho^{(1)}, f^{(1)}, v^{(1)}$ and $T^{(1)}$ at $N$ discrete points. Along most of the parameters domain, convergence of the calculation was established within $N \approx 50$. An increasing number of collocation points was required to capture the thin layer confinement of the convection layer, occurring when compressibility effects are dominant (see Sec. IV).

\section{Numerical scheme: DSMC method}

The DSMC method proposed by Bird [19] is a stochastic particle method commonly applied for the analysis of rarefied gas flows. In the present work, we apply the DSMC method to obtain results 
at near-continuum conditions, and validate the linear-stability analysis in Sec. II B. Additionally, the numerical scheme is used to investigate the system behavior at supercritical conditions, where the linear description becomes invalid.

We adopt Bird's algorithm and assume hard-sphere interactions between the gas molecules. To follow with problem formulation, the boundaries are assumed fully diffuse, and with prescribed heat flux. In difference from traditional applications of the wall-interaction algorithm (including the walls fixed-temperature Rayleigh-Bénard setup), the boundary temperature is treated as unknown. Application of the heat-flux condition therefore requires modification of the conventional computational scheme.

In recent contributions by the authors [20,21], a noniterative procedure for the imposition of a heat-flux condition in a DSMC calculation has been presented. The algorithm was assigned in onedimensional single- and bidirectional setups. The two-dimensional Rayleigh-Bénard configuration requires additional subdivision of the computational domain into subcells in the $x$ direction. Description of the specific numerical procedure follows.

Consider the interaction of gas molecules with a stationary diffuse reflecting boundary at the $j$-th computational cell located about $x=x_{j}$. The distributions of tangential and normal velocities of the molecules reflected at time $t=t_{i}$ are

$$
v_{t}^{*}\left(t_{i}, x_{j}\right)=\frac{1}{\sqrt{2} \beta_{w}^{*}\left(t_{i}, x_{j}\right)} R_{G} \quad \text { and } \quad v_{n}^{*}\left(t_{i}, x_{j}\right)=\frac{1}{\beta_{w}^{*}\left(t_{i}, x_{j}\right)} \sqrt{-\ln \left(R_{U}\right)},
$$

respectively, where $R_{U} \in[0,1]$ and $R_{G} \in(-\infty, \infty)$ are uniformly and Gaussian-distributed random numbers, and $\beta_{w}^{*}\left(t_{i}, x_{j}\right)=\left[2 \mathcal{R}^{*} T_{w}^{*}\left(t_{i}, x_{j}\right)\right]^{-1 / 2}$. Assigning a heat-flux boundary condition, the value of $\beta_{w}^{*}\left(t_{i}, x_{j}\right)$ is a priori unknown at each timestep and should be determined. This is carried out via imposition of mass and energy balances over the incoming and reflected molecules, yielding

$$
\frac{1}{\beta_{w}^{*^{2}}\left(t_{i}, x_{j}\right)}=\left\{\frac{\mathcal{H}_{\mathrm{in}}^{*}+q_{w}^{*}}{\mathcal{M}_{\mathrm{in}}^{*}}-\frac{u_{g, \text { in }}^{*^{2}}}{8}\right\}_{\left(t_{i}, x_{j}\right)},
$$

where $\mathcal{M}_{\text {in }}^{*}\left(t_{i}, x_{j}\right), u_{g, \text { in }}^{*}\left(t_{i}, x_{j}\right)$, and $\mathcal{H}_{\text {in }}^{*}\left(t_{i}, x_{j}\right)$ mark the macroscopic mass flux, gas velocity, and energy flux per unit mass, respectively. Summing over all $N$ particles impacting the wall about given location and time fixes

$$
\mathcal{M}_{\mathrm{in}}^{*}\left(t_{i}, x_{j}\right)=\frac{N}{\Delta t^{*} \Delta x^{*}}, \quad u_{g, \text { in }}^{*}\left(t_{i}, x_{j}\right)=\frac{1}{N} \sum_{n=1}^{N} \xi_{x}^{*^{(n)}},
$$

and

$$
\mathcal{H}_{\mathrm{in}}^{*}\left(t_{i}, x_{j}\right)=\frac{1}{\Delta t^{*} \Delta x^{*}} \sum_{n=1}^{N} \frac{1}{2}\left\{\left[\xi_{x}^{*^{(n)}}-\frac{1}{2} u_{g, \text { in }}^{*}\left(t_{i}, x_{j}\right)\right]^{2}+\left(\xi_{y}^{*^{(n)}}\right)^{2}+\left(\xi_{z}^{*^{(n)}}\right)^{2}\right\},
$$

where $\Delta t^{*}$ and $\Delta x^{*}$ are the timestep duration and boundary cell size, respectively. Having obtained $\beta_{w}^{*}\left(t_{i}, x_{j}\right)$ at each $x_{j}$ along the boundary, the simulation can be followed to the next timestep.

To facilitate comparison between DSMC and slip-flow scheme predictions, the initial state for DSMC calculations was set equal to the reference pure conduction distribution obtained in Sec. II A, specifying the walls heat-flux magnitudes. Due to Knudsen layer effects, inevitable inaccuracies in the slip-flow calculation occur in the vicinity of the boundaries. Direct imposition of the slip-flow-computed heat-flux would then lead to a reference state different from the one calculated by the slip-flow scheme. To overcome this difficulty, the simulation was started by prescribing temperature-fixed boundary conditions, ensuring the desired reference state. After completing several computational time-steps, the "numerically real" heat-flux was obtained through inversion of Eq. (21). The boundary conditions were then switched to heat-flux conditions, and the simulation was followed to later times. 


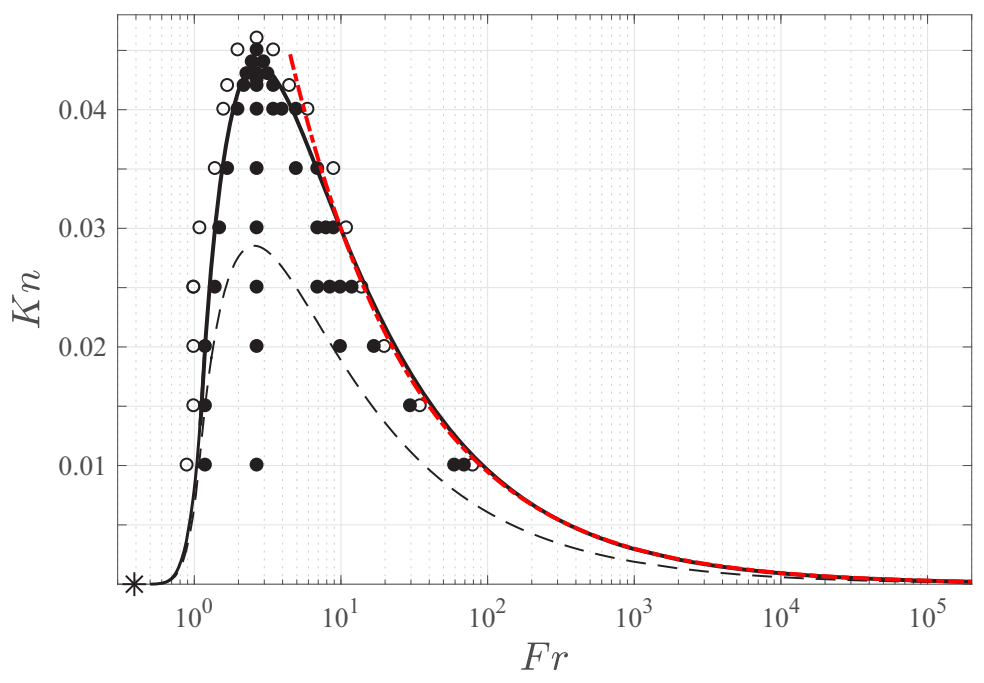

FIG. 1. Projection of the neutral surface $\omega(k ; \mathrm{Fr}, \mathrm{Kn})=0$ over the (Fr, Kn) plane at $R_{T}=0.1$. The solid line marks the neutral curve in the walls fixed heat-flux problem (along most of which $k \approx 0$; cf. the bold solid line in Fig. 2), and the dashed line shows the counterpart result in the isothermal walls setup (along most of which $k \approx 3.12$; cf. the thin dashed line in Fig. 2). The empty and filled circles denote states where the pure conduction solution is stable or unstable according to DSMC calculations, respectively, in the walls constant heat-flux problem. The red dash-dotted line marks the constant $\mathrm{Ra}_{m}=720$ large-Fr asymptote [see Eq. (23)], and the asterisk denotes the value $\mathrm{Fr}=\mathrm{Fr}_{\mathrm{cr}} \approx 0.39$, the minimal necessary Fr for instability at $\mathrm{Kn} \rightarrow 0$ [see Eq. (25)].

The DSMC calculations were performed in a rectangular computational domain with an aspect ratio of $L^{*} / D^{*}=2 \pi$. Periodic conditions were applied at the side $x^{*}=\mp L^{*} / 2$ boundaries. These conditions confine the spectrum of perturbation wave numbers to a discrete collection of $k=1,2, \ldots$, in difference from the continuous spectrum considered in the linear stability analysis [cf. Eq. (13)]. The significance of this difference is considered in Sec. III [see Fig. 3(b) and its discussion]. The computational domain contained $N_{x} \times N_{y}=50 \times 30$ cells, where each cell was subdivided into subcells to meet with the molecular mean free path constraint [19]. The timestep was set to $3-8 \times 10^{-3} D^{*} / U_{\mathrm{th}}^{*}$, depending on the Knudsen number, and each simulation was ran for 5-20 $\times 10^{4}$ timesteps. To shorten simulation times, the hydrodynamic fields were calculated via averaging of the microscopic distributions at every $5 \times 10^{3}$ timesteps. The number of particles in each simulation was $5 \times 10^{6}$, and ensemble averaging was employed over 8 realizations of the stochastic scheme. The simulations were carried out on an Intel i-7 eight-cores machine, and a typical calculation lasted between $24-48 \mathrm{~h}$.

\section{THE THRESHOLD OF INSTABILITY}

The threshold of instability in the heat-flux prescribed problem is now characterized. To this end, it is first noted that the linear scheme invariably yields real-valued $\omega$ (i.e., $\omega_{i}=0$ ) at all parameter combinations. The onset of instability therefore takes place via "exchange of stabilities," characterized by stationary perturbations [1]. This is in accordance with the results in the Boussinesq limit of the problem [12]. Focusing on a temperature ratio of $R_{T}=0.1$, Figs. 1 and 2 describe the projections of the neutral surface $\omega(k ; \mathrm{Fr}, \mathrm{Kn})=0$ onto the ( $\mathrm{Fr}, \mathrm{Kn})$ and $(\mathrm{Fr}, k)$ planes, respectively. The choice of $R_{T}=0.1$ is made to facilitate comparison with existing theoretical and numerical results for the walls fixed-temperature problem $[6,8]$. 


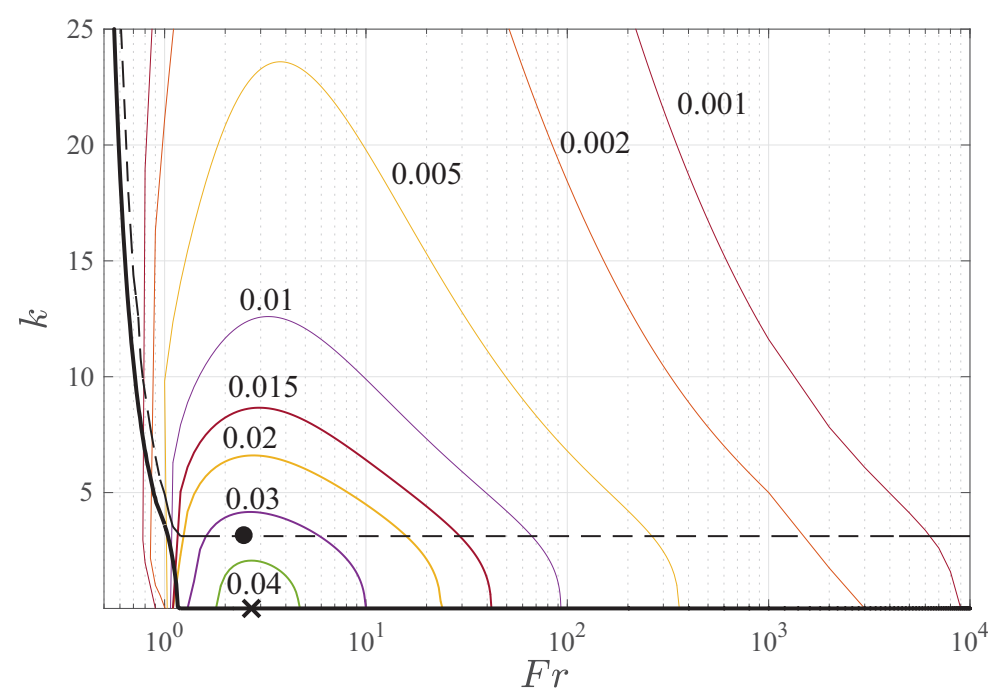

FIG. 2. Projection of the neutral surface over the $(\mathrm{Fr}, k)$ plane for $R_{T}=0.1$. The bold solid and thin dashed curves show projections of the neutral curves in Fig. 1 for the constant heat-flux and isothermal walls setups, respectively, where the cross and circle mark the respective peak $(\overline{\mathrm{Fr}}, \overline{\mathrm{Kn}})$ locations. The colored contours show the neutral curves at the indicated values of $\mathrm{Kn}$ in the constant heat-flux problem.

The bold solid line in Fig. 1 divides the (Fr, Kn) plane into domains of stable and unstable responses (above and below the line, respectively). This is compared with the dashed thin line, showing the counterpart result in the isothermal walls problem. Each point along these curves is characterized by a critical value of the wave number $k$, which fixes the wavelength of perturbations at the onset of instability, to be described in Fig. 2 (see the bold solid and thin dashed lines therein). Also appearing in Fig. 1 are the large-Fr asymptote, marked by the dash-dotted line, and corresponding to a constant value of the modified Rayleigh number [see Eq. (23) et seq.]; the asterisk at the lower left end of the neutral curve, denoting the minimal value of Fr required for instability at $\mathrm{Kn} \rightarrow 0$ [see Eq. (24) et seq.]; and DSMC predictions, depicted by filled and empty circles, for parameter combinations where instability occurs or not, respectively. It is viewed that, for each value of $\mathrm{Kn}$, the instability domain is confined to a Froude number interval of $\mathrm{Fr}_{\min } \leqslant \mathrm{Fr} \leqslant \mathrm{Fr}_{\max }$. The extent of the interval is rapidly decreasing with $\mathrm{Kn}$, vanishing above $\mathrm{Kn}=\overline{\mathrm{Kn}} \approx 0.0438$, where the reference state turns stable for all Fr according to linear stability calculations. The overall agreement between the slip-flow and DSMC results supports the present application of the former to analyze the problem. Slight discrepancies occur at parameter combinations in the vicinity of the peak $(\overline{\mathrm{Fr}}, \overline{\mathrm{Kn}})$ combination (where the DSMC scheme predicts a somewhat higher $\overline{\mathrm{Kn}} \approx 0.0452$ value), and along the lower right branch of the curve. In the former, these discrepancies may be attributed to the relatively nonsmall $\mathrm{Kn}$ values, which, combined with the large hydrodynamic gradients in the vicinity of the confining walls, lead to local Knudsen numbers where the slip-flow scheme is less effective. Along the lower right branch of the curve, the DSMC scheme predicts $\mathrm{Fr}_{\text {max }}$ values that are slightly lower than their counterpart linear-stability predictions. Notably, at these lower $\mathrm{Fr}_{\max }$ values, vanishingly small growth rates $\left[\omega_{r} \lesssim O\left(10^{-3}\right)\right]$ are obtained by linear stability calculations. Perturbations characterized by such small growth rates may be easily obscured by the statistical noise inherent in DSMC data. Given the equivocal nature of DSMC predictions at these conditions, and the matching between the slip-flow and Boussinesq-predicted threshold at large Fr (see below), we find the general agreement between the schemes satisfactory.

Focusing on the large-Fr branch of the neutral curve, we find that it is well approximated by the thin dash-dotted line $\mathrm{FrKn}^{2} \approx 8.98 \times 10^{-3}$, which, following the definition by Golshtein and 
Elperin [4], corresponds to a modified Rayleigh number of

$$
\mathrm{Ra}_{m}=\frac{2048}{75 \pi\left(1+R_{T}\right)^{2}} \frac{1-R_{T}}{\mathrm{FrKn}^{2}}=720 .
$$

This indicated value is identical with the critical Rayleigh number obtained in the Boussinesq limit of the problem [12], which further supports the accurateness of our calculations. Indeed, in the largeFr limit, compressibility effects become negligible, the pressure turns nearly uniform $\left[p^{(0)} \approx C\right.$, as inferred from Eq. (10)], and $\rho \approx \rho(T)$.

Considering the left branch of the neutral curve, we recall the necessary condition for instability in a compressible fluid, requiring that the adiabatic expansion of a fluid element rising through the reference pressure field should reduce its density below the ambient reference value [22]. In the present formulation, this yields the condition

$$
\frac{d T^{(0)}}{d y}+\frac{2(\gamma-1)}{\gamma \mathrm{Fr}}<0,
$$

which needs to be satisfied throughout, or at least along part, of the layer. Substituting $T^{(0)}(y)$ in Eq. (10) with $\mathrm{Kn} \rightarrow 0$ into Eq. (24), the minimal Froude number required for instability is

$$
\mathrm{Fr}_{\mathrm{cr}}=\frac{6 R_{T}^{1 / 2}}{5\left(1-R_{T}^{3 / 2}\right)},
$$

which yields, for the present $R_{T}=0.1, \mathrm{Fr}_{\mathrm{cr}} \approx 0.39$. This value, denoted by the asterisk in Fig. 1 , coincides with the lower left point of the calculated neutral curve. The onset of instability in this limit is discussed in separate in Sec. IV.

Comparing between the neutral curve in the walls constant heat-flux (bold solid line) and isothermal (thin dashed curve) setups, we observe a significant destabilizing effect of the former, extending the instability domain to a higher $\overline{\mathrm{Kn}}$, and widening the interval of unstable Froude numbers for each Kn. This agrees with the results in the Boussinesq limit, showing that the critical Rayleigh number in the constant heat-flux problem, $\mathrm{Ra}_{\mathrm{cr}}=720$, is smaller than in the isothermal walls setup, being $\mathrm{Ra}_{\mathrm{cr}} \approx 1708$ [1].

To further assess the effect of thermal boundary conditions on the threshold of instability, Fig. 2 presents a projection of the slip-flow calculated neutral surface on the $(\mathrm{Fr}, k)$ plane for $R_{T}=0.1$. The projections of the neutral curves in Fig. 1 are shown by the bold solid and thin dashed lines, and correspond to the constant heat-flux and isothermal walls setups, respectively. The colored contours further show the neutral curves at the indicated values of $\mathrm{Kn}$ in the constant heat-flux problem.

The bold solid and thin dashed lines in Fig. 2 show the variations of the critical wave number $k_{\mathrm{cr}}$ at the onset of instability along the neutral surfaces. Thus, the onset of instability along the high-Fr branch in the walls constant heat-flux setup is characterized by infinitely long $\left(k_{\mathrm{cr}}=0\right)$ waves, in accordance with the Bouusinesq-limit result $[12,13]$. This is qualitatively different from the high-Fr result in the isothermal walls configuration, where $k_{\mathrm{cr}} \approx 3.12$ [1]. While $k_{\mathrm{cr}}$ remains nearly constant along the right branch and the upper left parts of the neutral curves, it increases sharply for $\mathrm{Fr}_{\min } \lesssim 1$, in both setups. As will be discussed in Sec. IV, this non-Boussinesq effect is accompanied by the narrowing of the convection layer as $\mathrm{Fr}_{\min } \rightarrow \mathrm{Fr}_{\mathrm{cr}}$. The colored contours in Fig. 2 further confine the interval of wave numbers that are unstable at a given combination of $\mathrm{Fr}$ and $\mathrm{Kn}$. The contours are symmetric about $k=0$, hence only the $k>0$ half-plane is shown. Notably, the curvature of the contours is smaller along the left branch of the neutral surface compared with the high-Fr part. Consequently, any slight increase in $\mathrm{Fr}>\mathrm{Fr}_{\min }$ at a given $\mathrm{Kn}$ renders a wide range of perturbations unstable. As the Knudsen number approaches $\mathrm{Kn}=\overline{\mathrm{Kn}}$, the $(k, \mathrm{Fr})$ neutral curve is confined to a narrower interval of wave numbers in the vicinity of the critical wave number $\bar{k}=0$, corresponding to the peak location $(\overline{\mathrm{Fr}}, \overline{\mathrm{Kn}})$. For $\mathrm{Kn}>\overline{\mathrm{Kn}}$, the reference state turns stable for all $k$, as also inferred from Fig. 1. 

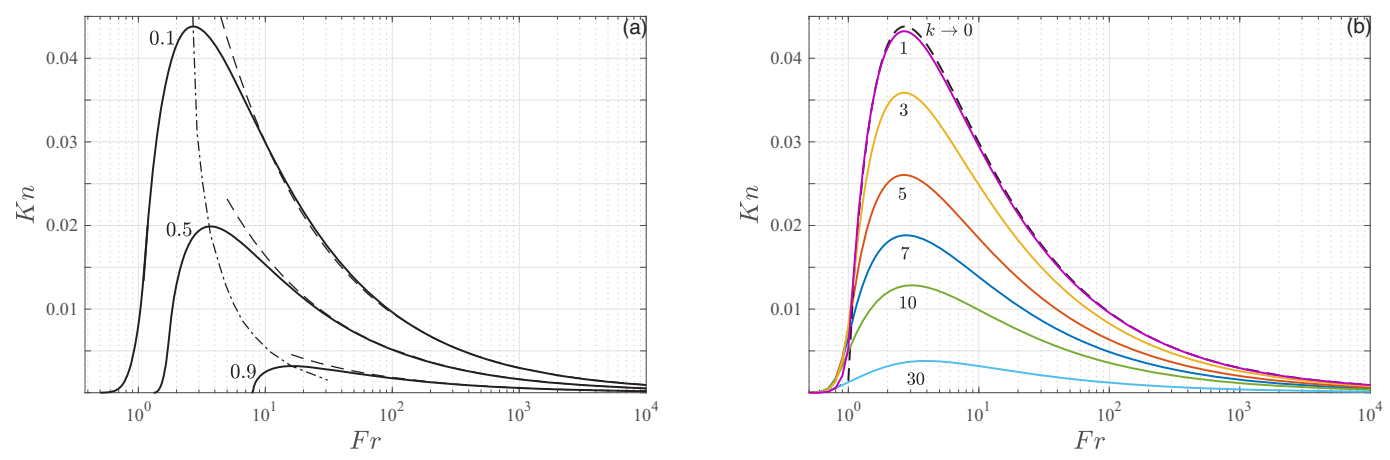

FIG. 3. Effects of the (a) temperature ratio $R_{T}$ and (b) perturbation wave number $k$ on the instability domain in the constant heat-flux problem. In Fig. 3(a), the numbers correspond to the values of $R_{T}$, the dashed lines mark the constant $R a_{m}$ asymptotes in each case, and the dash-dotted curve joins the locations of the neutral curve peaks ( $\overline{\mathrm{Fr}}, \overline{\mathrm{Kn}}$ ) at different $R_{T}$. In Fig. 3(b), $R_{T}=0.1$, and the numbers indicate the values of $k$ corresponding to each curve.

The effect of the temperature ratio $R_{T}$ on the instability threshold is presented in Figs. 3(a), where the reduction in the instability domain with decreasing $q_{w}$ is demonstrated. This is noted by a gradual decrease in $\overline{\mathrm{Kn}}$ and increase in $\overline{\mathrm{Fr}}$. The critical Boussinesq $\mathrm{Ra}_{m}=720$ value remains unchanged with varying $R_{T}$, and the variation in the dashed lines shapes is due to the different values of $R_{T}$, as seen from Eq. (23). Importantly, even for arbitrarily small temperature differences $\left(R_{T} \rightarrow 1\right)$, both Boussinesq and non-Boussinesq branches of the neutral surface coexist, confining instability to increasingly lower $\mathrm{Kn} \rightarrow 0$ [23].

The impact of the perturbation wave number $k$ on the onset of instability is examined in Fig. 3(b). The figure presents the $(\mathrm{Fr}, \mathrm{Kn})$ projections of the neutral surface at the indicated values of the wave number $k$. This is relevant for assessing the effect of considering a discrete $k=1,2, \ldots$ wave-number spectrum on the results in DSMC calculations, as opposed to the continuum spectrum analyzed in the linear stability scheme (see Sec. IIC). While the change from a continuous to a discrete spectrum might have resulted in a reduction in the convection domain, the results in Fig. 3(b) indicate that the $k \rightarrow 0$ curve only slightly differs from the $k=1$ line. The agreement obtained in Fig. 1 between the computations is therefore rationalized. With increasing $k$, instability sets in at lower Knudsen numbers, apart from the lower left branch, where shorter waves turn unstable at higher $\mathrm{Kn}$ (observe the intersections between the constant $k$ curves as $\mathrm{Fr} \rightarrow \mathrm{Fr}_{\mathrm{cr}}$ ). This is further discussed in Sec. IV, where the onset of instability in the compressible limit is analyzed.

The effect of the thermal boundary conditions on the perturbations flow field at the onset of instability is studied in Fig. 4. The figure presents the critical eigenfunctions for the tangential velocity and temperature at $R_{T}=0.1$. Figures 4(a) and 4(b) compare between the constant heatflux and isothermal walls results at the respective peak $(\overline{\mathrm{Fr}}, \overline{\mathrm{Kn}})$ locations, whereas Figs. 4(c) and $4(\mathrm{~d})$ show similar comparison at $(\mathrm{Fr}, \mathrm{Kn})=\left(\mathrm{Fr}_{\min }, 0.005\right)$ combinations along the low-Fr branch of the neutral curve (see Fig. 1). At first we note the considerable effect of boundary conditions on the temperature perturbations, compared with the seemingly minor impact on the tangential velocity. The latter, nevertheless, represent convection cells that differ distinctly due to the difference in the associated critical wave numbers $k_{\mathrm{cr}}$. Specifically, for the heat-flux-imposed setup, $k_{\mathrm{cr}}=0$ and $\approx 3.4$ at $(\mathrm{Fr}, \mathrm{Kn})=(\overline{\mathrm{Fr}}, \overline{\mathrm{Kn}})$ and $\left(\mathrm{Fr}_{\min }, 0.005\right)$, respectively, whereas $k_{\mathrm{cr}} \approx 3.12$ and $\approx 4.7$ at the counterpart isothermal-walls conditions (cf. Fig. 2). This results in wider (and, for $k_{\mathrm{cr}}=0$, infinitely long) convection cells in the heat-flux-prescribed configuration. Considering the temperature eigenfunctions in Figs. 4(b) and 4(d), the effect of different thermal conditions is clearly viewed, impacting the temperature perturbation across the entire layer. In particular, the heat-flux conditions result in temperature variations that are larger in amplitude at the $y=0.5$ 

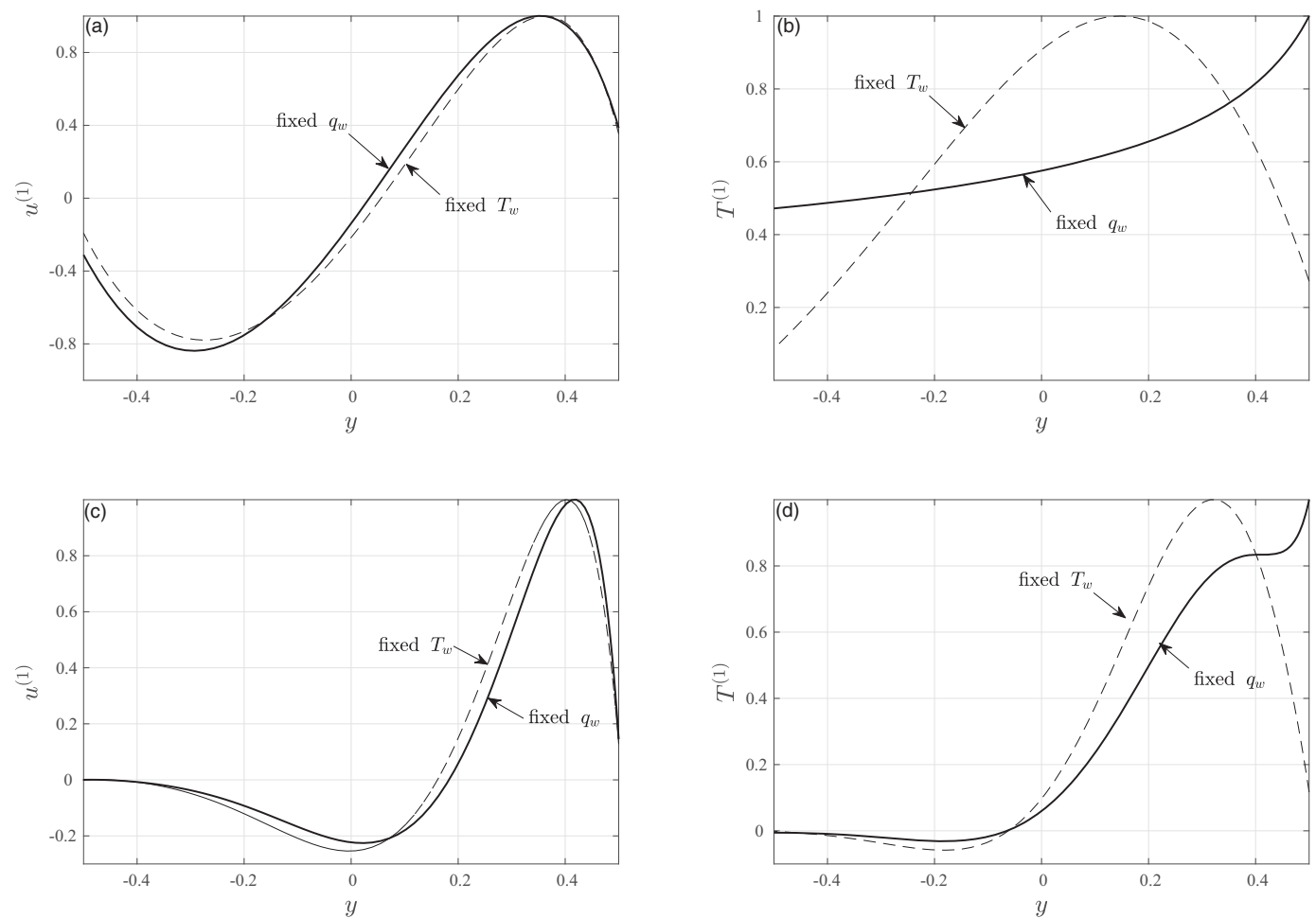

FIG. 4. The critical tangential velocity (a), (c) and temperature (b), (d) eigenfunctions at the onset of instability for $R_{T}=0.1$ : comparison between walls constant heat-flux (solid lines) and isothermal boundaries (dashed curves) setups at $(\mathrm{Fr}, \mathrm{Kn})=(\overline{\mathrm{Fr}}, \overline{\mathrm{Kn}})(\mathrm{a}),(\mathrm{b})$ and $(\mathrm{Fr}, \mathrm{Kn})=\left(\mathrm{Fr}_{\min }, 0.005\right)(\mathrm{c}),(\mathrm{d})$.

cold wall. In common to both setups, the convection layer, occupying the entire gas expanse at $(\mathrm{Fr}, \mathrm{Kn})=(\overline{\mathrm{Fr}}, \overline{\mathrm{Kn}})$, becomes more confined to the cold wall vicinity at $(\mathrm{Fr}, \mathrm{Kn})=\left(\mathrm{Fr}_{\min }, 0.005\right)$, where compressibility effects turn dominant. This is further analyzed in the next section.

\section{THE LIMIT OF DOMINANT COMPRESSIBILITY}

Focusing on the limit of dominant compressibility, we analyze the system instability in the vicinity of the lower left end of the neutral curve in Fig. 1, for which

$$
\mathrm{Fr}=\operatorname{Fr}_{\mathrm{cr}}(1+\delta),
$$

with $\delta \ll 1$ and $\mathrm{Kn} \rightarrow 0$. The analysis follows the scheme presented in Ref. [9] for the isothermal walls setup, and we therefore outline it briefly for completeness.

We seek for a limit case solution that predicts the thinning of the convection layer to the vicinity of the upper cold wall as $\mathrm{Fr} \rightarrow \mathrm{Fr}_{\mathrm{cr}}$. Toward this end, we set $\omega=0$ in Eqs. (14)-(17), and introduce the scaling

$$
k=l \delta^{-1} \quad \text { and } \quad \mathrm{Kn}=a \delta^{5 / 2},
$$

where $a$ and $l$ are assumed $\sim O(1)$. The hydrodynamic perturbations are consequently expanded via

$$
\begin{aligned}
& f^{(1)} \approx f_{0}^{(1)}+\delta f_{1}^{(1)}+\ldots, \quad v^{(1)} \approx \delta\left(v_{0}^{(1)}+\delta v_{1}^{(1)}+\ldots\right), \\
& \rho^{(1)} \approx \delta^{3 / 2}\left(\rho_{0}^{(1)}+\delta \rho_{1}^{(1)}+\ldots\right) \quad \text { and } T^{(1)} \approx \delta^{3 / 2}\left(T_{0}^{(1)}+\delta T_{1}^{(1)}+\ldots\right) .
\end{aligned}
$$


In search for a nontrivial solution at the upper $\frac{1}{2}-y \sim O(\delta)$ layer, an inner coordinate

$$
Y=l\left(\frac{1}{2}-y\right) \delta^{-1}
$$

is introduced. The system of equations is then rewritten in terms of $Y$, to form an inner problem that vanishes at the layer far edge. The leading-order system of equations may be reduced to a single equation for the normal velocity,

$$
\left(\frac{d^{2}}{d \eta^{2}}-A_{0}^{-2 / 7}\right)^{3} v_{0}^{(1)}=\eta v_{0}^{(1)}
$$

where

$$
\eta=A_{0}^{1 / 7}\left(Y-B_{0} / A_{0}\right)
$$

and

$$
A_{0}=\frac{16 \alpha}{75 a^{2}\left(\operatorname{Fr}_{\mathrm{cr}} R_{T} l\right)^{5}}, \quad B_{0}=\frac{8 \alpha}{15 a^{2}\left(\operatorname{Fr}_{\mathrm{cr}} R_{T} l\right)^{4}}, \quad \alpha=\left[\frac{2 \mathrm{e}^{5}}{\mathrm{e}^{5 / R_{T}^{1 / 2}}-\mathrm{e}^{5}}\right]^{2} .
$$

Equation (30) is supplemented by the boundary conditions of impermeability, velocity-slip, and zero heat-flux at the cold wall,

$$
v_{0}^{(1)}=0, \quad \frac{d v_{0}^{(1)}}{d \eta}=0, \quad \text { and } \quad\left(\frac{d^{5}}{d \eta^{5}}-2 A_{0}^{-2 / 7} \frac{d^{3}}{d \eta^{3}}\right) v_{0}^{(1)}=0 \quad \text { at } \quad \eta=-B_{0} A_{0}^{6 / 7},
$$

respectively, together with a matching decay condition at $\eta \rightarrow \infty$. Applying complex-plane analysis, the general solution for Eq. (30) is given by

$$
v_{0}^{(1)}=\sum_{m=0}^{5} K_{n} f_{n}
$$

where $K_{n}$ are arbitrary complex constants, and

$$
f_{n}(\eta)=\int_{C_{n}} \exp \left[-\frac{1}{7} z^{7}+\frac{3}{5} A_{0}^{-2 / 7} z^{5}-A_{0}^{-4 / 7} z^{3}+A_{0}^{-6 / 7} z+\eta z\right] d z
$$

are generalized Laplace integrals in the complex $z$ plane. The contours $C_{n}$ originate and terminate at $z \rightarrow \infty$ within each of the sectors

$$
-\frac{\pi}{14}+\frac{2 \pi n}{7}<\arg z<\frac{\pi}{14}+\frac{2 \pi n}{7}, \quad n=0,1, \ldots, 6 .
$$

The eigenvalue problem yielding the desired dispersion relation is obtained by applying the decay and boundary conditions in Eq. (31) to Eq. (32), and requiring a nontrivial solution. At $\eta \rightarrow \infty$, the method of steepest descent yields the leading order estimate

$$
\begin{aligned}
f_{n} \approx & \sqrt{\frac{\pi}{3}} \eta^{-5 / 12} \exp \left[\frac{6}{7} \mathrm{e}^{\pi n i / 3} \eta^{7 / 6}+\frac{3}{5} \mathrm{e}^{-\pi n i / 3} A_{0}^{-2 / 7} \eta^{5 / 6}-\frac{1}{4} \mathrm{e}^{\pi n i} A_{0}^{-4 / 7} \eta^{1 / 2}\right. \\
& \left.+\frac{3}{8} \mathrm{e}^{\pi n i / 3} A_{0}^{-6 / 7} \eta^{1 / 6}-\frac{\pi n i}{6}\right] .
\end{aligned}
$$

The decay condition then fixes $K_{0}=K_{1}=K_{5}=0$. The remaining constants $K_{2}, K_{3}$, and $K_{4}$ are determined through Eq. (31), yielding the homogeneous system of linear algebraic 

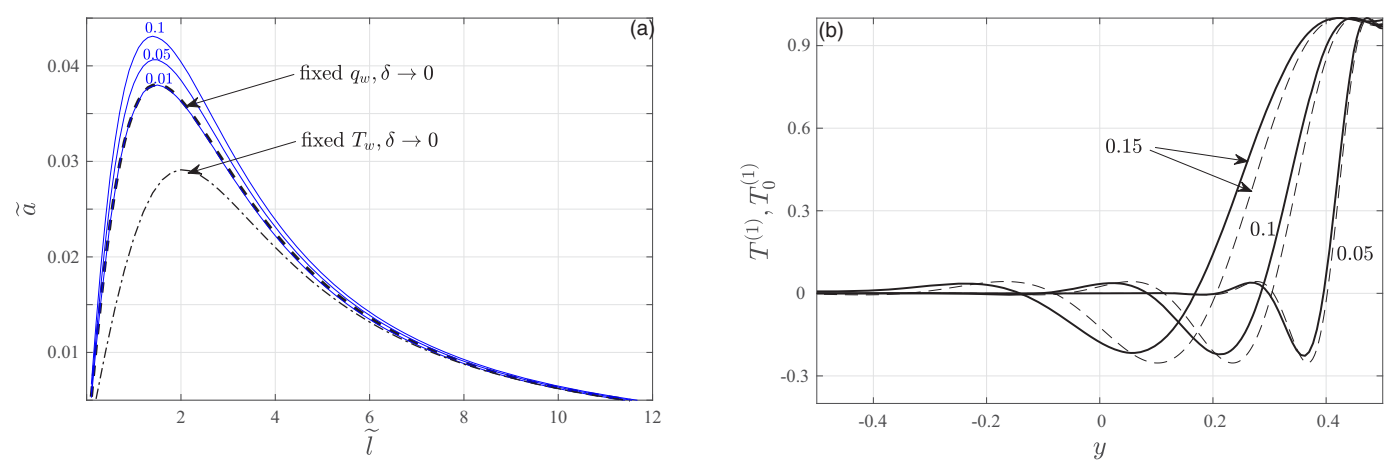

FIG. 5. (a) The neutral curve in the $(\tilde{l}, \widetilde{a})$ plane for $R_{T}=0.5$. The dashed and dash-dotted lines show the dispersion relation Eq. (37) in the limit $\delta \rightarrow 0$ in the constant wall heat-flux and fixed wall temperature problems, respectively, and the solid blue lines correspond to the numerically calculated neutral curves at the indicated values of $\delta$ in the former case. (b) The normalized temperature eigenfunctions in the constant wall heat-flux setup for $R_{T}=0.5$ and $\left(\widetilde{l}_{\mathrm{cr}}, \widetilde{a}_{\max }\right)$ combinations. The solid and dashed curves represent the slip-flow numerical $\left(T^{(1)}\right)$ and asymptotic $\left(T_{0}^{(1)}\right)$ solutions at the indicated values of $\delta$, respectively.

equations

$$
\begin{aligned}
& K_{2}\left(I_{3}^{0}-I_{2}^{0}\right)+K_{3}\left(I_{4}^{0}-I_{3}^{0}\right)+K_{4}\left(I_{4}^{0}-I_{5}^{0}\right)=0, \\
& K_{2}\left(I_{3}^{1}-I_{2}^{1}\right)+K_{3}\left(I_{4}^{1}-I_{3}^{1}\right)+K_{4}\left(I_{4}^{1}-I_{5}^{1}\right)=0, \quad \text { and } \\
& K_{2}\left[I_{3}^{5}-I_{2}^{5}-2 A_{0}^{-2 / 7}\left(I_{3}^{3}-I_{2}^{3}\right)\right]+K_{3}\left[I_{4}^{5}-I_{4}^{5}-2 A_{0}^{-2 / 7}\left(I_{4}^{3}-I_{4}^{3}\right)\right]+K_{4}\left[I_{4}^{5}-I_{5}^{5}-2 A_{0}^{-2 / 7}\left(I_{4}^{3}-I_{5}^{3}\right)\right] \\
& \quad=0,
\end{aligned}
$$

where

$$
I_{n}^{p}=e^{(p+1) i \theta_{n}} \int_{0}^{\infty} r^{p} \exp \left[G_{n}(r)\right] d r
$$

and

$$
G_{n}(r)=-\frac{1}{7} r^{7} e^{7 i \theta_{n}}+\frac{3}{5} A_{0}^{-2 / 7} r^{5} e^{5 i \theta_{n}}-A_{0}^{-4 / 7} r^{3} e^{3 i \theta_{n}}+A_{0}^{-6 / 7} r e^{i \theta_{n}}\left(1-B_{0}\right)
$$

A nontrivial solution for the above set of equations exists when its characteristic determinant vanishes. Numerical evaluation of the determinant terms yields the dispersion relation $A_{0}=A_{0}\left(B_{0}\right)$, which is more conveniently represented by

$$
\widetilde{a}=\tilde{a}(\tilde{l})
$$

with

$$
\tilde{l}=B_{0} / A_{0} \quad \text { and } \quad \tilde{a}=A_{0}^{2} / B_{0}^{5 / 2} .
$$

In difference from the analysis in Ref. [9], the present calculation applies a zero heat-flux condition in Eq. (31) [given explicitly by the third equation in Eq. (36)] in place of a temperature jump condition. The effect of this difference on the results is now examined. Notably, in this limit of particularly small Knudsen numbers, DSMC runs become prohibitively expensive, and we therefore present only slip-flow-based results. We focus on the case $R_{T}=0.5$, for which $\mathrm{Fr}_{\mathrm{cr}} \approx 1.31$ [see Eq. (25)].

Figure 5 presents the convergence with $\delta$ of the numerical solution described in Sec. II B to the current $\delta \rightarrow 0$ approximation. Figure 5(a) shows the convergence of the numerically calculated neutral curve to Eq. (37) in the $(\widetilde{l}, \widetilde{a})$ plane, and compares between the walls constant heat-flux and isothermal setups. Figure 5(b) demonstrates the convergence of the temperature eigenfunction 

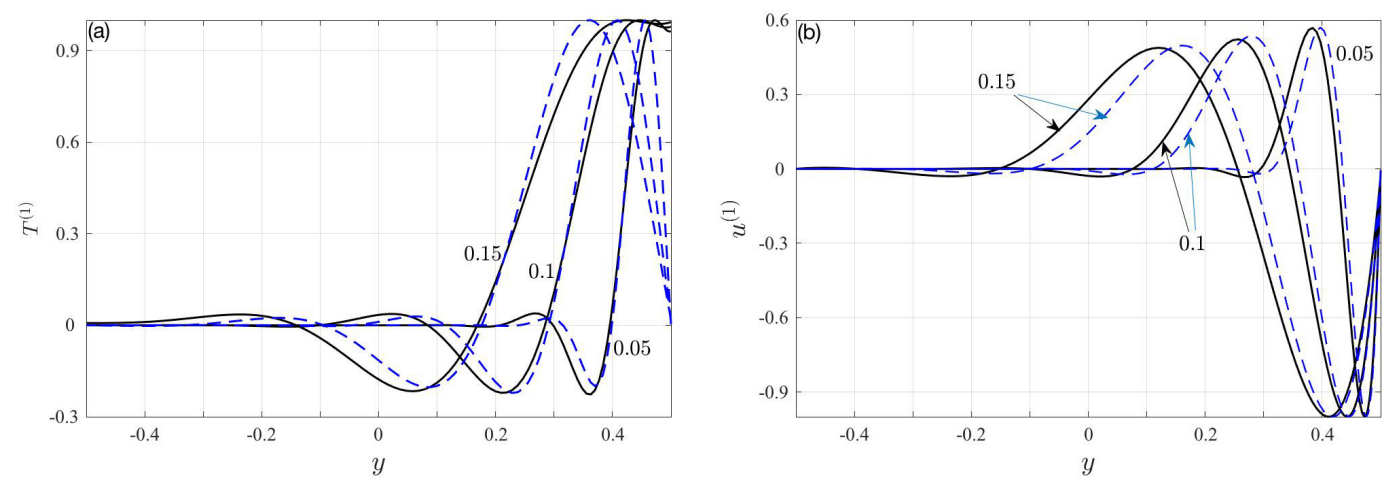

FIG. 6. The effect of thermal boundary conditions on the (a) temperature and (b) vertical velocity perturbation eigenfunctions in the compressible limit. In each part, the solid and dashed curves present the normalized eigenfunctions for the walls constant heat-flux and fixed temperature problems, respectively, at the indicated values of $\delta$.

to the asymptotic result with decreasing $\delta$. In agreement with the above discussion, the results in Fig. 5(a) indicate the destabilizing effect of the heat-flux conditions, yielding an extended instability domain. The thinning of the convection layer with decreasing $\delta$ is presented in Fig. 5(b). The layer confinement is directly correlated with a reduction in the layer width where the necessary condition for instability Eq. (24) is satisfied [9]. The numerical and asymptotic results nearly coincide at $\delta=0.05$, and the differences become indiscernible at lower $\delta$.

Similar to the result in Fig. 4(d), the temperature perturbation at the $y=0.5$ wall satisfies the adiabatic condition in Eq. (19). This is qualitatively different from the isothermal walls setup, where $T^{(1)}$ vanishes at the wall for $\mathrm{Kn} \rightarrow 0$. The difference is illustrated in Fig. 6(a), where the temperature eigenfunctions are compared between the two cases. The asymptotic solution agrees with the result in Fig. 4(d), indicating that temperature perturbation variations become largest at the cold wall vicinity in the heat-flux prescribed setup. Quantitative differences are observed between the vertical velocity eigenfunctions $v^{(1)}$ in Fig. 6(b), where the constant heat-flux conditions result in a somewhat wider convection layer at a given $\delta$.

\section{NONLINEAR REGIME}

We now consider the system behavior at supercritical conditions, corresponding to parameter combinations that are within the instability domain mapped in Fig. 1. At these states, the linear stability scheme yields positive perturbation growth rates, for which the underlying small-amplitude assumption breaks down. The following discussion is therefore based on DSMC computations only, according to which, at the $0.01 \leqslant \mathrm{Kn}<\overline{\mathrm{Kn}}$ range simulated, a final steady periodic state is reached.

Figure 7 presents the effect of the Knudsen number, at fixed $R_{T}=0.1$ and $\mathrm{Fr}=2.7$, on the supercritical DSMC-calculated velocity and temperature perturbation fields. At each value of Kn, a comparison is made between the walls constant heat-flux [Figs. 7(a), 7(c) and 7(e)] and fixed temperature [Figs. 7(b), 7(d) and 7(f)] results. The $|\mathbf{u}|^{\max }$ values denote the maximum velocity amplitude obtained in each part. The temperature colormap range is kept identical between the setups at each $\mathrm{Kn}$, to visualize the effect of the different boundary conditions on the perturbation field strength.

At the largest $\mathrm{Kn}=0.04$ value presented, the isothermal-walls reference state is stable (see the dashed line in Fig. 1). Hence, both velocity and temperature perturbation fields in Fig. 7(b) exhibit "noisy" random values. In further agreement with Fig. 1, the same reference state is unstable when subject to constant heat-flux conditions, as shown by the distinct convection rolls in Fig. 7(a), dominated by a $k=2$ wave-number component. Decreasing the Knudsen number to 

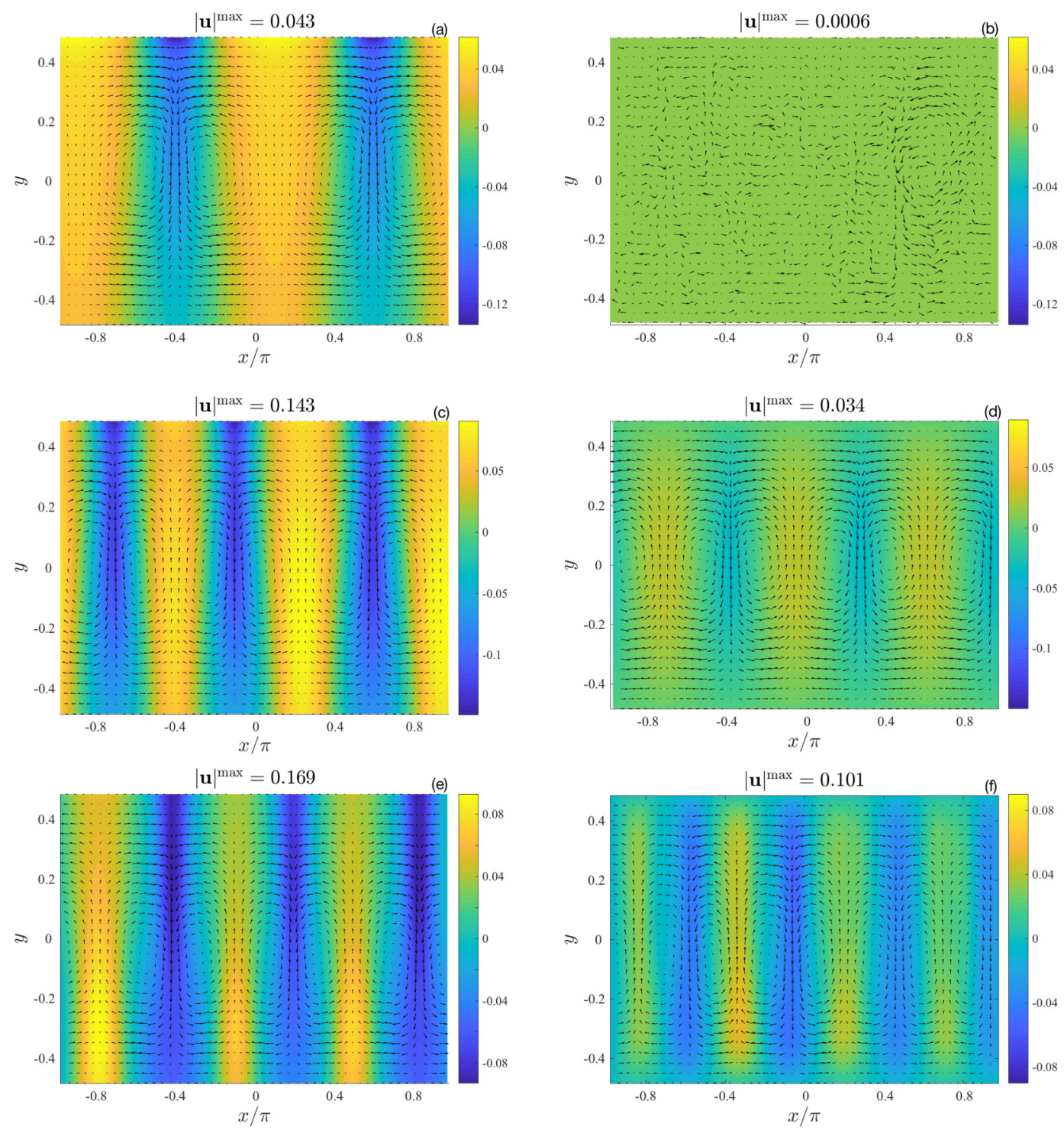

FIG. 7. DSMC-calculated flow fields (arrows) and temperature perturbations (colormaps) for walls constant heat-flux (a,c,e) and fixed temperature (b,d,f) setups at $R_{T}=0.1, \mathrm{Fr}=2.7$ and $\mathrm{Kn}=0.04$ $(\mathrm{a}, \mathrm{b}), \mathrm{Kn}=0.025(\mathrm{c}, \mathrm{d})$, and $\mathrm{Kn}=0.01(\mathrm{e}, \mathrm{f})$. In each part, $|\mathbf{u}|^{\max }$ denotes the maximum value of calculated velocity amplitude.

$\mathrm{Kn}=0.025$, the isothermal walls configuration becomes unstable as well, yet with a considerably weaker convection strength and temperature perturbation amplitude compared with the constant heat-flux setup [cf. Figs. 7(c) and 7(d)]. A similar trend is observed with decreasing Kn further to $\mathrm{Kn}=0.01$ in Figs. 7(e) and 7(f), where the flow fields in the isothermal and constant heat-flux wall setups are dominated by $k=3$ and $k=4$ wave-number components, respectively. Apart from the differences in convection amplitudes and patterns, the two setups vary in the forms of temperature perturbation fields. Specifically, while the temperature perturbation at the walls in the isothermal boundaries setup is vanishingly small (in accordance with the $O(\mathrm{Kn})$ jump condition) and reaches a maximum value in the bulk of the gas layer, it is monotonically varying with $y$ in the constant 


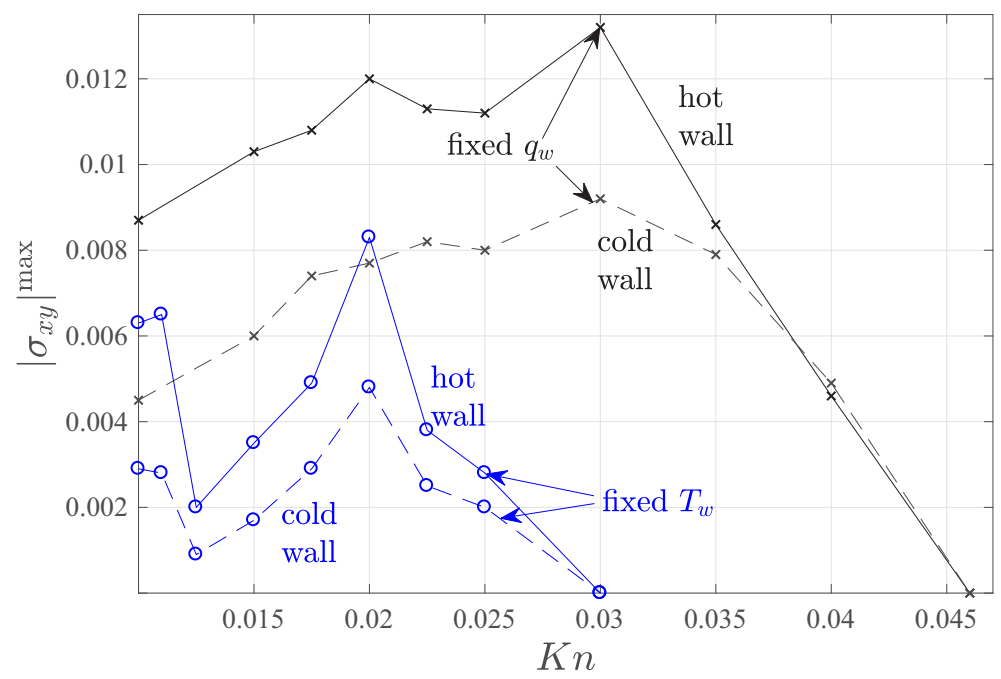

FIG. 8. Variation with Kn of the DSMC-calculated walls shear stress amplitude at $R_{T}=0.1$ and $\mathrm{Fr}=2.7$. Black and blue curves present results for walls constant heat-flux and isothermal setups, respectively, with solid and dashed lines showing shear-stress amplitudes at the hot and cold walls, respectively.

heat-flux setup, and obtains extremal values at the upper cold or lower hot walls. This result is qualitatively supported by the predictions in Fig. 4(b) for the critical temperature eigenfunctions at $(\mathrm{Fr}, \mathrm{Kn})=(\overline{\mathrm{Fr}}, \overline{\mathrm{Kn}})$.

A quantity of common interest in analyses of the RB problem at supercritical conditions is the walls shear stress, hereafter denoted by $\sigma_{x y}[24,25]$. Making use of the DSMC scheme, the stress is calculated by summing over the differences in molecules tangential momenta before and after wall collisions. Since all final states obtained in our calculations are $x$-periodic, the measure of interest at each parameter combination is the amplitude of shear stress variation, $\left|\sigma_{x y}\right|^{\max }$. Figure 8 presents the variation of $\left|\sigma_{x y}\right|^{\max }$ with $\mathrm{Kn}$ at $R_{T}=0.1$ and $\mathrm{Fr}=2.7$. The figure compares between the results in the walls heat-flux (black lines) and temperature prescribed (blue curves) setups at the hot (solid) and cold (dashed) boundaries. Distinctly, the stresses in the constant heat-flux setup are always larger than their isothermal-wall counterparts. This agrees with the stronger convection speeds observed in the former in Fig. 7, accompanied by larger velocity gradients at the walls. The shear stresses in each case vanish at Knudsen numbers above the respective $\mathrm{Kn}=\overline{\mathrm{Kn}}$ threshold (see the maxima points in the dashed and solid lines in Fig. 1). At a given configuration, the stress at the hot wall is larger than at the cold wall, reflecting mainly the larger value of gas viscosity at the higher temperature [see Eq. (7)]. Remarkably, the Kn variation in all cases is nonmonotonic and contains several maxima and minima points along the $0.01 \leqslant \mathrm{Kn} \leqslant \overline{\mathrm{Kn}}$ interval. While the eventual decrease in $\left|\sigma_{x y}\right|^{\max }$ as $\mathrm{Kn} \rightarrow \overline{\mathrm{Kn}}$ results from the stabilizing effect of gas rarefaction and consequent decrease in convection speed, its increase with Kn at sufficiently lower $\mathrm{Kn}<\overline{\mathrm{Kn}}$ reflects the amplifying effect of rarefaction on the boundary shear stress at constant shear rate [21]. Noting the characteristic dimensional shear rate by $u^{*} / D^{*}$ (where $u^{*}$ marks the characteristic convection speed), it is therefore a balance between the simultaneous decrease in $u^{*}$ and $D^{*}$ with $\mathrm{Kn}$ (for a fixed mean free path $l^{*}$ ) that determines the nonmonotonic behavior observed in Fig. 8.

\section{CONCLUSION}

We considered the effect of heat-flux boundary conditions, replacing the previously studied isothermal boundary conditions, on the Rayleigh-Bénard instability in a rarefied gas. The problem was investigated in the limit of small Knudsen numbers, by means of a linear stability analysis 
of a near-continuum slip flow model, together with the direct simulation Monte Carlo method. In the latter, a noniterative algorithm was applied to implement the heat-flux conditions. The results obtained delineate the instability domain in the plane of parameters of the Knudsen number, Froude number and walls reference temperature ratio. The heat-flux conditions were shown to have a considerable destabilizing effect, extending instability to higher Knudsen numbers. Asymptotic analysis of the limit where gas compressibility is dominant and convection is confined to a narrow layer was carried out. The Monte Carlo scheme was applied to investigate the system instability at supercritical conditions, where the heat-flux conditions result in elevated flow stresses. Nonmonotonic variations in the walls shear stresses with Kn were observed. These were rationalized in terms of the countering effects of gas rarefaction, which reduces convection velocities and associated gradients, yet increases the shear force at constant shear rate due to the reduction in system size.

The present work complements previous studies on the Rayleigh-Bénard instability in rarefied gases, all dedicated to the problem at isothermal walls conditions. Having demonstrated the impact of a change in the thermal boundary conditions on the system properties, it may be of interest to consider the effect of heat-flux conditions in other canonical stability problems, including the Taylor-Couette [26] or Kolmogorov [27] setups. As the dynamic and thermodynamic fluid descriptions are inevitably coupled at noncontinuum states, thermal conditions modifications may prove effective in monitoring these and other instability phenomena in rarefied gases. Further investigations on this topic are currently underway.

\section{ACKNOWLEDGMENTS}

This research was supported by the Israel Science Foundation (Grant No. 1084/16) and by the Aeronautical Engineering Research Fund. Y.B. acknowledges the support by the Adams Fellowship Program of the Israel Academy of Sciences and Humanities.

[1] S. Chandrasekhar, Hydrodynamic and Hydromagnetic Stability (Clarendon, Oxford, 1961).

[2] E. Koschmieder, Bénard Cells, and Taylor Vortices (Cambridge University Press, Cambridge, 1993).

[3] E. Bodenschatz, W. Pesch, and G. Ahlers, Recent developments in Rayleigh-Bénard convection, Annu. Rev. Fluid Mech. 32, 709 (2000).

[4] E. Golshtein and T. Elperin, Convective instabilities in rarefied gases by direct simulation monte carlo method, J. Thermophys. Heat Transfer 10, 250 (1996).

[5] Y. Sone, K. Aoki, and H. Sugimoto, The Bénard problem for a rarefied gas: Formation of steady flow patterns and stability of array of rolls, Phys. Fluids 9, 3898 (1997).

[6] S. Stefanov, V. Roussinov, and C. Cercignani, Rayleigh-Benard flow of a rarefied gas and its attractors. I. Convection regime, Phys. Fluids 14, 2255 (2002).

[7] S. Stefanov, V. Roussinov, and C. Cercignani, Rayleigh-Bénard flow of a rarefied gas and its attractors. II. Chaotic and periodic convective regimes, Phys. Fluids 14, 2270 (2002).

[8] A. Manela and I. Frankel, On the Rayleigh-Bénard problem in the continuum limit, Phys. Fluids 17, 036101 (2005).

[9] A. Manela and I. Frankel, On the Rayleigh-Bénard problem: Dominant compressibility effects, J. Fluid Mech. 565, 461 (2006).

[10] J. Zhang and J. Fan, Monte Carlo simulation of thermal fluctuations below the onset of Rayleigh-Bénard convection, Phys. Rev. E 79, 056302 (2009).

[11] J. Zhang and T. Onskog, Langevin equation elucidates the mechanism of the Rayleigh-Bénard instability by coupling molecular motions and macroscopic fluctuations, Phys. Rev. E 96, 043104 (2017).

[12] E. M. Sparrow, R. J. Goldstein, and V. K. Jonsson, Thermal instability in a horizontal fluid layer: Effect of boundary conditions and non-linear temperature profile, J. Fluid Mech. 18, 513 (1964). 
[13] E. Jakeman, Convective instability in fluids of high thermal diffusivity, Phys. Fluids 11, 10 (1968).

[14] C. J. Chapman, S. Childress, and M. R. E. Proctor, Long wavelength thermal convection between nonconducting boundaries, Earth Planet. Sci. Lett. 51, 362 (1980).

[15] H. Park and L. Sirovich, Hydrodynamic stability of Rayleigh-Bénard convection with constant heat-flux boundary conditions, Q. Appl. Math. 49, 313 (1991).

[16] Y. Sone, Molecular Gas Dynamics: Theory, Techniques, and Applications (Birkhäuser, Boston, 2007).

[17] K. Aoki, C. Baranger, M. Hattori, S. Kosuge, G. Martalo, J. Mathiaud, and L. Mieussens, Slip boundary conditions for the compressible Navier-Stokes equations, J. Stat. Phys. 169, 744 (2017).

[18] R. Peyret, Spectral Methods for Incompressible Viscous Flow (Springer, New York, 2002).

[19] G. Bird, Molecular Gas Dynamics and the Direct Simulation of Gas Flows (Clarendon, Oxford, 1994).

[20] Y. Ben Ami and A. Manela, Acoustic field of a pulsating cylinder in a rarefied gas: Thermoviscous and curvature effects, Phys. Rev. Fluids 2, 093401 (2017).

[21] Y. Ben Ami and A. Manela, Nonlinear thermal effects in unsteady shear flows of a rarefied gas, Phys. Rev. E 98, 033121 (2018).

[22] L. Landau and E. Lifshitz, Fluid Mechanics (Pergamon, Reading, MA, 1959).

[23] A. Manela and I. Frankel, From the generalized Boussinesq approximation to the marginally superadiabatic limit, Chem. Eng. Commun. 197, 51 (2010).

[24] M. Reeuwijk, H. J. J. Jonker, and K. Hanjalić, Wind and boundary layers in Rayleigh-Bénard convection. I. Analysis and modeling, Phys. Rev. E 77, 036311 (2008).

[25] F. Chilla and J. Schumacher, New perspectives in turbulent Rayleigh-Bénard convection, Eur. Phys. J. E 35, 58 (2016).

[26] A. Manela and I. Frankel, On the compressible Taylor-Couette problem, J. Fluid Mech. 588, 59 (2007).

[27] A. Manela and J. Zhang, The effect of compressibility on the stability of wall-bounded Kolmogorov flow, J. Fluid Mech. 694, 29 (2012). 